\title{
Influences of partial components in firefighters' personal protective equipment on subjective perception
}

\author{
Siyeon Kim", Young Joon Jang ${ }^{1}$, Yoon Jeong Baek ${ }^{1}$ and Joo-Young Lee ${ }^{2}$
}

\author{
* Correspondence: yeulmia@snu.ac.kr \\ ${ }^{1}$ Department of Textiles, \\ Merchandising and Fashion Design, \\ Seoul National University, Seoul, \\ Korea \\ Full list of author information is \\ available at the end of the article
}

\begin{abstract}
The purpose of the present study was to investigate the influences of partial components of firefighters' personal protective equipment (PPE) on subjective perception during exercise and recovery at $28^{\circ} \mathrm{C}, 40 \% \mathrm{RH}$. Eight firefighters participated in eight conditions consisting of various combinations of PPE components weighing from 7.8 to $14.9 \mathrm{~kg}$ (fully equipped with all PPE, FullPPE; FullPPE without a self-contained breathing apparatus, NoSCBA; FullPPE without a helmet, NoHelmet; FullPPE without gloves, NoGloves; FullPPE without boots, NoBoots; Full PPE without bunker jacket or pants, NoJP; Not equipped with a helmet, gloves, boots, or breathing apparatus, NoHGBS and Control (1.1 kg; wearing shorts, long-sleeved shirts and long pants)). The results showed: 1) Alleviation of subjectively perceived strain was significantly greater in NoJP during both exercise and recovery than NoHelmet and Nogloves, while the NoJP showed no significant difference with NoBoots and NoHGBS. 2) NoBoots was significantly effective in relieving physiological thermal burden rather than in psychological strain. 3) Exposing the hands and feet affected the local thermal sensation and sweat sensation of the hands and feet, respectively, while exposing the head did not affect local head thermal sensation and sweat sensation. 4) The relationship between clothing microclimate humidity and sweat sensation showed a logarithmic function. The present study indicate that weight loss in boots alleviated physiological strain during exercise, whereas after exercise, doffing jacket and taking off gloves, boots, and helmet simultaneously were both most effective way to relieve thermal strain quickly.
\end{abstract}

Keywords: Personal protective equipment (PPE); Thermal sensation; Sweat sensation; Protective boots; Clothing weight

\section{Introduction}

Firefighters encounter a range of physiological burden when dealing with tasks under conditions of high environmental heat stress (Barr et al. 2010; Rossi 2003). Not only do they experience an increased metabolic rate resulting from performing physical tasks such as fire suppressing, searching, and rescuing with burdensome equipment (Barr et al. 2010; Gledhill and Jamnik 1992; Qu and Yeo 2011) but they also face disrupted heat dissipation induced by flame-resistant fabric and multi-layered clothing (Rossi 2003). Although the personal protective equipment (PPE) contribute to efficient protection against hazardous environments, studies have shown the existence of high

\section{Springer}

(c) 2014 Kim et al.; licensee Springer. This is an Open Access article distributed under the terms of the Creative Commons Attribution License (http://creativecommons.org/licenses/by/2.0), which permits unrestricted use, distribution, and reproduction in any medium, provided the original work is properly cited. 
cardiorespiratory and thermal strain along with feelings of discomfort in hazardous environments requiring the use of impermeable protective clothing (McLellan et al. 1999, Son et al. 2013; Turpin-Legendre and Meyer 2007; White et al. 1989).

In an effort to relieve firefighters' physiological burden, various methods including lessening the weight of total PPE (Griefahn et al. 2003; Hooper et al. 2001), using ventilation (Turpin-Legendre and Meyer 2007), and active cooling with ice cooling vest or water (Barr et al. 2009; Barr et al. 2011; Chou et al. 2008) were widely studied. In particular, using devices for cooling or ventilation could result in both increased weight of clothing, which induced additional physiological and physical strain, and increased cost of firefighting gear. For this reason, studies rating the efficiency of various body parts for applying cooling or ventilating are needed.

Taylor et al. (2012) explored the fractional contributions of each part of the firefighter's protective gear in order to detect the segment of clothing which significantly mitigates physiological burden. The results showed that footwear induced the greatest relative metabolic impact during walking and bench stepping, being 8.7 and 6.4 times greater per unit mass than the breathing apparatus. The study found that the most efficient way to reduce the physiological burden of firefighters' protective equipment would be to reduce the mass of the boots. Reducing the boots' mass can effectively alleviate wearers fatigue along with metabolic rate (Blacker et al. 2010; Knapik et al. 2004; Lee et al. 2013; Taylor et al. 2012) since the feet are known as one of the most inefficient locations for load carriage as well was the hands (Lind and McNicol 1968).

Most studies have investigated the physiological strain of firefighters due to the PPE or evaluated physiological responses along with psychological responses which have been supportive information. However, very few studies focused on the subjective measures regarding firefighters' PPE and still no published research is available that investigates subjective perception for evaluating the fractional influence of PPE. Subjectively perceived sensations of heat play a key role in thermoregulatory behavior through a feed forward system, resulting in a delay of autonomic thermoregulation (Bligh 1973). Several studies explored the impact factors for thermal comfort in neutral environments (Frank et al. 1998), various environments (Gagge et al. 1967), or during exercise (Winslow et al. 1939). Significant correlations between thermal sensation and rectal temperature were reported (White et al. 1989), Thermal comfort showed a strong correlation with skin wetness (Gagge and Gonzalez 1974; Havenith et al. 2002).

Therefore, the purpose of this study was to investigate firefighters' various subjective perceptions including thermal sensation, thermal comfort, sweat sensation, thirst sensation, and rating of perceived exertion (RPE) while wearing a wide range of PPE combinations. Total sweat rate, clothing microclimate humidity and blood lactate concentration were evaluated as the supportive measures of subjective perceptions. We hypothesized that reducing the mass of footwear (or removing boots) would be more effective in relieving psychological strain as well than reducing the load carried by the head, trunk, or hands.

\section{Methods}

\section{Subjects}

Eight professional male firefighters participated in the study [mean \pm SD: $39.4 \pm 5.6$ years in age, $173.9 \pm 3.8 \mathrm{~cm}$ in height, $74.2 \pm 10.0 \mathrm{~kg}$ in body mass, $1.88 \pm 0.13 \mathrm{~m}^{2}$ in body surface area, $20.2 \pm 7.4 \%$ body fat (\%BF), $42.0 \pm 5.1 \mathrm{ml} \cdot \mathrm{kg}^{-1} \cdot \mathrm{min}^{-1}$ in maximal oxygen 
consumption $\left(V \mathrm{O}_{2 \max }\right), 197 \pm 6 \mathrm{bpm}$ in maximal heart rate $\left(\mathrm{HR}_{\max }\right)$, and $10.4 \pm 7.0$ years of employment]. Prior to obtaining written informed consent, the subjects were all informed of the content, purpose, and potential risks of the present study. This study was approved by the Institutional Review Board of Seoul National University (IRB \#1209/ 001-001).

Each firefighter visited nine times at the same time of a day for the $V \mathrm{O}_{2 \max }$ test and eight experimental trials. They were encouraged to refrain from alcohol use and strenuous exercise for $48 \mathrm{~h}$, along with food and caffeine for $3 \mathrm{~h}$ prior to each trial. On such occasions that emergency called for firefighting, the experimental schedule of the firefighter was rescheduled to keep above regulations. Each visit was separated by at least $48 \mathrm{~h}$ while the eight trials were completed over a span of four weeks. To avoid the effect of familiarization during the eight tests, the experiments were scheduled across subjects according to the Latin square method.

\section{Experimental design and procedures}

To evaluate the impact of each component of the personal protective equipment, eight clothing conditions were designed by removing one or several parts of the clothing ensemble (Table 1): Full PPE (14.9 kg in total mass of PPE), NoSCBA (7.8 kg), NoHelmet (13.6 kg), NoGloves (14.8 kg), NoBoots (12.8 kg), NoJP (11.7 kg), NoHGBS (11.4 kg), and Control $(1.1 \mathrm{~kg})$. We used the SCBA without gas. For the NoBoots and NoHGBS conditions, firefighters wore indoor sleepers. The face-piece of the SCBA was replaced by a respiratory mask connected with a gas exchange analyzer in order to collect respiratory gases. Total PPE mass did not include the mass of the mask (172 g) used to collect respiratory gases.

Air temperature and relative humidity were maintained at $28^{\circ} \mathrm{C}$ and $40 \% \mathrm{RH}$, respectively. Prior to commencing procedures subjects drank $300 \mathrm{ml}$ water. Each test began with resting on a chair for stabilization $(10 \mathrm{~min})$ followed by a treadmill exercise at $5.5 \mathrm{~km} \cdot \mathrm{hr}^{-1}$ (with $1 \%$ slope) for $30 \mathrm{~min}$. Upon cessation of exercise they rested on a stool for $20 \mathrm{~min}$ for recovery. The test was terminated if their rectal temperature

Table 1 Characteristics of eight experimental conditions of fire fighter's personal protective equipment

\begin{tabular}{|c|c|c|c|}
\hline Experimental conditions & Common items & Differential items & $\begin{array}{l}\text { Total garment } \\
\text { mass }(\mathbf{k g})\end{array}$ \\
\hline 1. Control & & Slippers & 1.128 \\
\hline 2. Full PPE & & $\begin{array}{l}\text { Socks, Bunker jacket and pants, Hood, } \\
\text { Helmet, Gloves, Boots, SCBA }\end{array}$ & 14.937 \\
\hline 3. No SCBA & & $\begin{array}{l}\text { Socks, Bunker jacket and pants, Hood, } \\
\text { Helmet, Gloves, Boots }\end{array}$ & 7.793 \\
\hline 4. No helmet & & $\begin{array}{l}\text { Socks, Bunker jacket and pants, Gloves, } \\
\text { Boots, SCBA }\end{array}$ & 13.643 \\
\hline 5. No gloves & $\begin{array}{l}\text { Shorts, Shirts (long), } \\
\text { Pants (long) }\end{array}$ & $\begin{array}{l}\text { Socks, Bunker jacket and pants, Hood, } \\
\text { Helmet, Boots, SCBA }\end{array}$ & 14.750 \\
\hline 6. No boots & & $\begin{array}{l}\text { Slippers, Bunker jacket and pants, Hood, } \\
\text { Helmet, Gloves, SCBA }\end{array}$ & 12.830 \\
\hline 7. No Jacket/Pants (NoJP) & & Socks, Hood, Helmet, Boots, SCBA & 11.658 \\
\hline $\begin{array}{l}\text { 8. No Helmet/Gloves/ } \\
\text { Boots/SCBA (NoHGBS) }\end{array}$ & & Slippers, Bunker jacket and pants & 11.418 \\
\hline
\end{tabular}


reached $39.2^{\circ} \mathrm{C}$, their heart rate $(\mathrm{HR})$ reached $95 \%$ of their maximal $\mathrm{HR}$, or if any volunteer was unable to continue the exercise. Immediately after completing the session the subjects' experimental clothing was removed.

\section{Measurements and calculations}

Before and after each trial, subjects who only wore undershorts and shorts were weighed on a calibrated scale (ID2, Mettler-Toledo, Germany; resolution of 1 gram). Also, all experimental garments were weighed before and after each trial to estimate absorbed sweat volume.

To evaluate subjects' thermal sensation, sweat sensation, thermal comfort, and thirst sensation, subjects were asked to update their condition every 10 minutes over a period of an hour. As a form of measurement, a 9-point categorical scale was used for thermal sensation and a 7-point categorical scale for sweat sensation, thermal comfort, and thirst sensation. Ratings of perceived exertion (RPE) ranging from 'light (6)' to 'extremely hard (20)' (Borg 1982) were also asked of subjects every 10 min during exercise.

Capillary blood samples were collected from the tip of the middle finger at rest and 3 min after the cessation of exercise to measure blood lactate concentration. The blood samples were analyzed using a portable lactate analyzer (Lactate Pro ${ }^{\mathrm{m} /} / \mathrm{LT} 1710$, Arkray KDK Corporation, Japan). Total sweat rate (TSR) was calculated from differences in body weight between the beginning and end of the trial. Absorbed sweat rate (ASR) was calculated from the difference of total clothing masses between the beginning and end of the trial. Using TSR and ASR, evaporated sweat rate (ESR) was calculated as well. Clothing microclimate humidity was measured on the middle of the chest in the most inner layer between long-sleeved shirts and the skin using a portable thermohygrograph (Thermo Recorder TR-72U, Japan) every 5 s. Perceived wetted body regions were asked every $10 \mathrm{~min}$, and perceived wetness area was calculated according to the method from Lee et al. (2008).

\section{Data analyses}

All data were expressed as the mean for the final 3 min of each period including standard deviation (mean $\pm \mathrm{SD}$ ). One-way analyses of variance (ANOVA) with repeated measures were used to identify differences in physiological responses among the eight conditions. The Duncan's post-hoc test was used to assess the parameters that displayed significant differences in ANOVA. All statistical analyses were performed with SPSS 18.0. Significance level was set at $P<0.05$.

\section{Results}

\section{Overall subjective perceptions}

During the exercise period, all conditions except for NoJP and Control showed no significant difference in thermal sensation. NoJP were perceived to be cooler than other PPE conditions and the responses between NoJP and Control showed no significant differences (Figure 1A). While subjects recovered, differences among the conditions were more distinguishable. In particular, the standard deviation of thermal sensation for FullPPE in recovery was approximately three times greater than that during exercise. Thermal sensations were quickly recovered into the neutral states in NoJP and NoHGBS, showing a statistically similar level as Control $(P<0.05)$ (Figure $1 \mathrm{~A})$. 

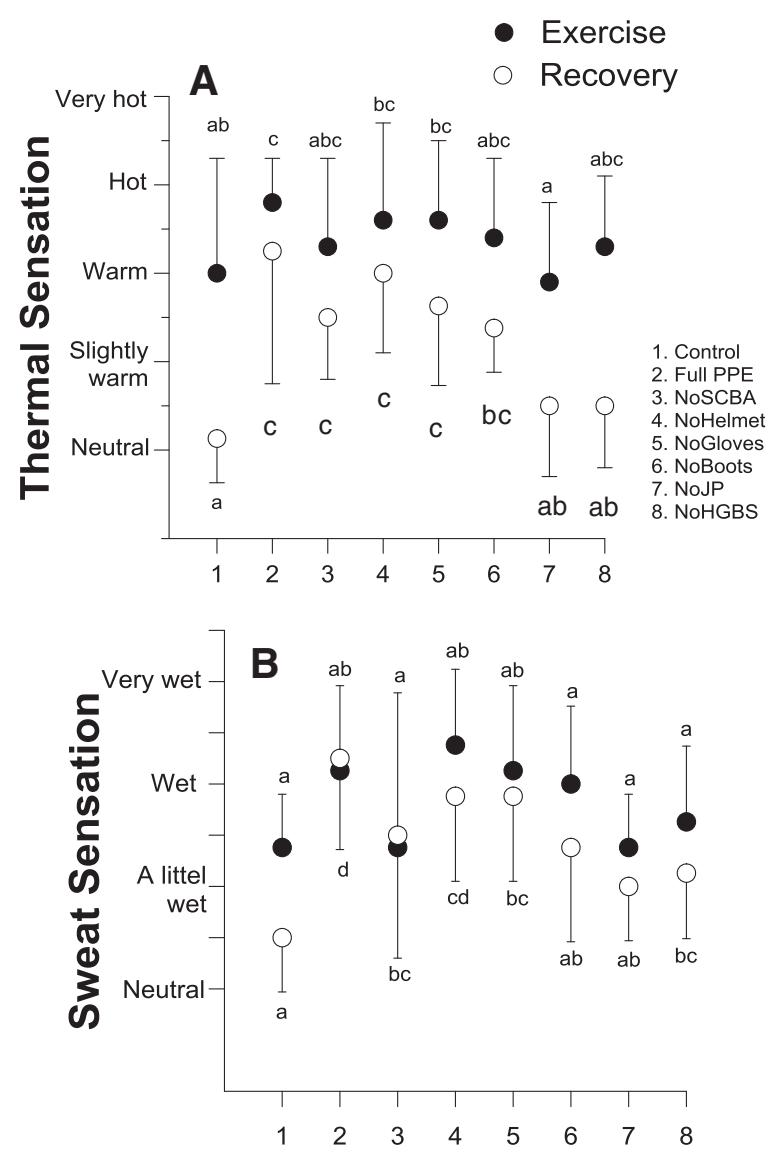

Figure 1 Comparisons between eight conditions for thermal sensation (A) and sweat sensation (B) during exercise and recovery. Closed circles $(\bullet)$ and opened circles ( 0 ) represent values during exercise at $38^{\text {th }}$ min and recovery at $58^{\text {th }}$ min respectively. The letters $(a, b, c, d)$ above error bars and below error bars represent significant differences among eight conditions during exercise and recovery,

respectively as a result of Duncan analysis $(P<0.05)$.

As for sweat sensation at the end of exercise subjects expressed the most sweat sensation in NoHelmet, FullPPE, and NoGloves with no significant differences (Figure 1B). During the recovery, subjects expressed feeling less wet compared to the end of exercise, but in FullPPE and NoSCBA sweat sensation showed no significant differences between the end of exercise and in the recovery period (Figure 1B).

With regard to thermal comfort, subjects felt more discomfort in order followed; FullPPE, NoHelmet, NoGloves, NoBoots, NoSCBA, NoHGBS, NoJP and Control, though there was no significant difference except for thermal comfort in Control, which was significantly lower than in FullPPE (Table 2). Thirst sensation during recovery was significantly lower in NoJP and NoHGBS than in FullPPE $(P<0.05$; Table 2).

Ratings of perceived exertion at the end of exercise $\left(\mathrm{RPE}_{\max }\right)$ were the greatest in the FullPPE $(14.6 \pm 1.8)$ and NoHelmet $(14.6 \pm 2.1)$, followed by NoSCBA $(13.5 \pm 1.9)$ and NoGloves $(13.5 \pm 1.7)(P<0.05)$. On the other hand, RPE in NoBoots $(13.25 \pm 1.28)$, NoJP $(12.50 \pm 2.14)$, and NoHGBS $(13.13 \pm 1.73)$ were statistically lower than those in FullPPE and NoHelmet $(P<0.05$; Figure 2$)$. 
Table 2 Subjective responses, sweat rate and clothing microclimate humidity for the firefighters

\begin{tabular}{|c|c|c|c|c|c|c|c|c|}
\hline \multirow[t]{2}{*}{ Parameter } & \multicolumn{8}{|c|}{ Experimental condition $\mathrm{N}=8$} \\
\hline & 1. Control & 2. Full PPE & 3. No SCBA & 4. No helmet & 5. No gloves & 6. No boots & 7. No jacket/pants (NoJP) & $\begin{array}{l}\text { 8. No helmet/gloves/ } \\
\text { boots/SCBA (NoHGBS) }\end{array}$ \\
\hline Thermal comfort in exercise & $-0.50 \pm 1.07^{*}$ & $-1.75 \pm 1.16$ & $-1.38 \pm 1.06$ & $-1.75 \pm 1.16$ & $-1.50 \pm 1.07$ & $-1.50 \pm 1.31$ & $-0.88 \pm 0.64$ & $-1.13 \pm 0.99$ \\
\hline Thirst sensation in recovery & $1.0 \pm 0.9$ & $1.6 \pm 0.7$ & $1.1 \pm 0.6$ & $1.2 \pm 0.6$ & $1.2 \pm 0.6$ & $1.0 \pm 0.6$ & $0.8 \pm 0.6^{*}$ & $0.9 \pm 0.8^{*}$ \\
\hline Total sweat rate (g) & $282 \pm 83^{* * *}$ & $743 \pm 248$ & $711 \pm 301$ & $696 \pm 206$ & $767 \pm 308$ & $600 \pm 174$ & $456 \pm 131^{* *}$ & $532 \pm 139$ \\
\hline Sweat rate_evaporated (g) & $273 \pm 76$ & $377 \pm 69$ & $363 \pm 109$ & $403 \pm 301$ & $382 \pm 192$ & $329 \pm 75$ & $377 \pm 124$ & $355 \pm 86$ \\
\hline Sweat rate_absorbed (g) & $10 \pm 8^{* * *}$ & $366 \pm 192$ & $384 \pm 197$ & $293.4 \pm 170$ & $385 \pm 145$ & $270 \pm 138$ & $79 \pm 48^{* * *}$ & $177 \pm 70^{* *}$ \\
\hline Evaporation rate $(\%)^{1)}$ & $96.9 \pm 2.0^{* * *}$ & $52.5 \pm 7.8$ & $52.7 \pm 6.2$ & $55.0 \pm 22.4$ & $49.8 \pm 9.7$ & $56.4 \pm 12.7$ & $82.6 \pm 9.0^{* * *}$ & $67.4 \pm 6.6^{* *}$ \\
\hline Absorption rate $(\%)^{2)}$ & $3.1 \pm 2.0^{* * *}$ & $47.5 \pm 7.8$ & $47.3 \pm 6.2$ & $45.0 \pm 22.4$ & $50.2 \pm 9.7$ & $43.6 \pm 12.7$ & $17.4 \pm 9.0^{* * *}$ & $32.6 \pm 6.6^{* *}$ \\
\hline Humidity in clothing at rest (\%) & $39.0 \pm 5.8^{*}$ & $50.5 \pm 11.0$ & $38.5 \pm 9.5^{*}$ & $38.2 \pm 6.4^{*}$ & $43.6 \pm 7.5$ & $47.4 \pm 4.3$ & $37.7 \pm 8.1^{* *}$ & $41.2 \pm 8.9^{*}$ \\
\hline Humidity in clothing in exercise (\%) & $80.6 \pm 4.7^{* * *}$ & $95.4 \pm 4.5$ & $85.0 \pm 7.6^{* * *}$ & $94.6 \pm 4.0$ & $94.1 \pm 4.8$ & $95.9 \pm 2.2$ & $87.0 \pm 8.3^{* *}$ & $94.0 \pm 5.0$ \\
\hline Humidity in clothing in recovery (\%) & $75.8 \pm 9.2^{*}$ & $99.0 \pm 0.1$ & $97.8 \pm 1.8$ & $98.7 \pm 0.5$ & $99.0 \pm 0.0$ & $98.5 \pm 1.3$ & $84.5 \pm 10.4^{* * *}$ & $98.2 \pm 1.0$ \\
\hline Time to 99\%RH in clothing microclimate ( $\mathrm{min}$ ) & - & 32 & 38 & 35 & 33 & 33 & 23 & 35 \\
\hline Number of person who reached 99\%RH & 0 & 8 & 5 & 7 & 8 & 7 & 1 & 4 \\
\hline Covered area (\%) & 0 & 100 & 100 & 95.1 & 95.1 & 93.2 & 20 & 83.4 \\
\hline Perceived wetted area in exercise (\%) & $18.3 \pm 13.5^{* * *}$ & $71.2 \pm 29.2$ & $63.8 \pm 30.5$ & $69.7 \pm 30.1$ & $56.7 \pm 34.4$ & $47.7 \pm 23.3^{*}$ & $29.7 \pm 11.3^{* * *}$ & $60.0 \pm 24.6$ \\
\hline Perceived wetted area in recovery (\%) & $6.9 \pm 12.6^{* * *}$ & $70.9 \pm 31.6$ & $65.0 \pm 29.9$ & $69.1 \pm 32.4$ & $50.6 \pm 35.2$ & $51.0 \pm 25.6$ & $13.4 \pm 18.7^{* * *}$ & $57.6 \pm 31.7$ \\
\hline
\end{tabular}

(Thermal comfort: -2 (Uncomfortable), -1 (A little uncomfortable), 0 (Not both), Thirst sensation: 0 (No thirsty), 1 (A little thirsty), 2 (Thirsty); ${ }^{1}$ Evaporation rate (\%) $=(\text { Evaporated sweat rate }(\mathrm{g}) / \text { Total sweat rate }(\mathrm{g}))^{*} 100$, ${ }^{2)}$ Absorption rate $\left.(\%)=(\text { Absorbed sweat rate }(\mathrm{g}) / \text { Total sweat rate }(\mathrm{g}))^{*} 100\right)$; ${ }^{*}$ Significantly different to FullPPE $(P<0.05),{ }^{*}$ Significantly different to FulllPPE $(P<0.01)$, ***Significantly different to FullPPE $(P<0.001)$. 


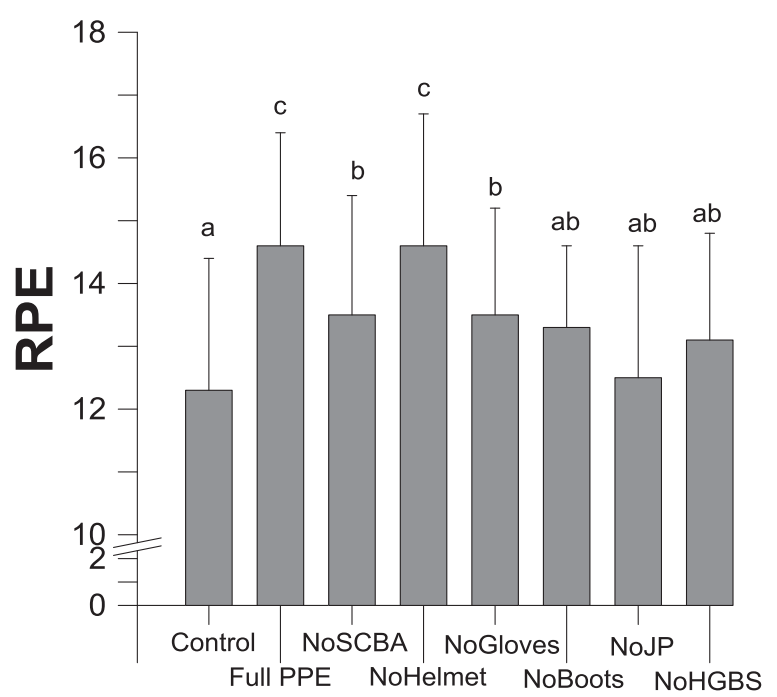

Figure 2 Ratings of perceived exertion at the end of exercise $\left(\mathrm{RPE}_{\max }\right)$. Different alphabets mean that they are significantly different groups by Duncan post-hoc test $(P<0.05)$.

\section{Local subjective perceptions}

At the end of exercise, local thermal sensation on all body regions was not significantly different between FullPPE and NoHelmet. Among the eight conditions, results from three single PPE conditions (NoHelemt, NoGloves, and NoBoots) with FullPPE were shown in Figure 3. Thermal sensation during the recovery period was lower, especially in the exposed body regions in NoGloves and NoBoots, while for the NoHelmet condition head thermal sensation did not show any differences from thermal sensation on other body regions (Figure 3).

Local sweat sensation showed more distinct difference between values in exercise and recovery than regional thermal sensation (Figure 3). In FullPPE, local sweat sensation around the head and hands tended to be greater than those on other regions. In NoGloves, sweat sensation on the hands tended to be lower than those on other body regions, and sweat sensation around the feet in NoBoots tended to be lower than those on other regions, while for the NoHelmet condition, head sweat sensation did not reflect any benefit of the head exposure.

\section{Total sweat rate and clothing microclimate humidity}

Total sweat rate $($ TSR $)$ was lower in NoJP $(P<0.01)$ and in NoHGBS $(P=0.053)$ than in FullPPE (Table 2). While all conditions showed no statistical difference in evaporated sweat rate (ESR), evaporation rate (\%) was apparently higher in Control (97\%), NoJP (83\%), and NoHGBS (67\%) compared to FullPPE (53\%) $(P<0.01)$. Absorbed sweat rate (ASR) was also significantly lower in Control, NoJP, NoHGBS and so was the \% absorption rate. Mean values of absorption rates in Full PPE, NoSCBA, NoHelmet, NoGloves, NoBoots ranged from about $44 \%$ to $50 \%$. ASR was the highest in NoGloves (385 g per trial) and FullPPE (366 g per trial). Among these five conditions, the lowest value of ASR was $270 \mathrm{~g}$ in NoBoots (Table 2).

Clothing microclimate humidity showed significant differences among the eight conditions during rest, exercise and recovery periods. In Control, No SCBA, NoHelmet, 


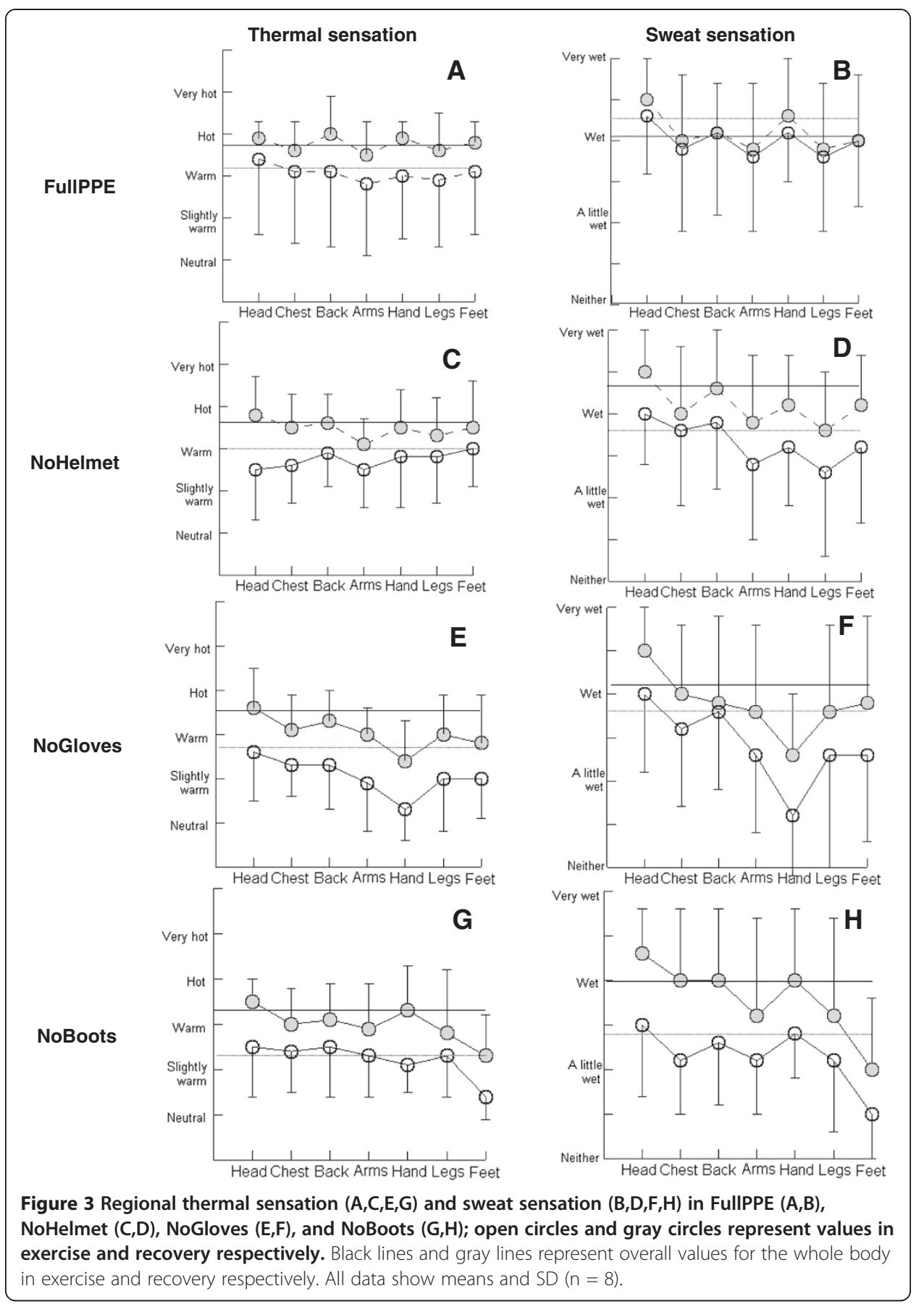

NoJP, and NoHGBS at rest clothing microclimate humidity on the chest was lower than that in FullPPE $(P<0.05)$. Around the end of exercise, clothing humidity on the chest for most conditions approached near 99\%RH (Table 2). For all subjects in Full PPE and NoGloves, the clothing humidity reached 99\%RH, seven subjects had reached this mark in NoHelmet and NoBoots, and only one subject in NoJP reached $99 \% \mathrm{RH}$ (Table 2). 


\section{Blood lactate concentration}

At the end of exercise, blood lactate concentration showed the greatest value in the condition of FullPPE (increased value $=1.69$ ) followed by NoSCBA, NoHGBS, NoHelmet, and NoGloves (Figure 4). Changes in blood lactate concentration in NoBoots and NoJP were lower than those in FullPPE, NoSCBA, and NoHGBS (Figure 4).

\section{Relationship between sweat sensation and microclimate humidity}

The relationship between sweat sensation and clothing microclimate humidity on the chest at the cessation of exercise over the eight conditions showed a logarithmic function (Figure 5). As the humidity inside clothing increased, sweat sensation gradually increased and showed a dramatic rise around the level of 95\%RH. In NoSCBA and NoJP, sweat sensations were maintained at the level of Control regardless of the differences of humidity in clothing, which indicates that the range of $80 \sim 88 \% \mathrm{RH}$ might induce a similar sweat sensation falling between score 1 (a little wet) and score 2 (wet). Among the other five conditions, subjects expressed lower sweat sensation in NoHGBS than in any other conditions.

\section{Relationships of evaporation with PPE total weight, exposed body surface area, and perceived skin wetness}

There were linear relationships between evaporation rate (\%) and PPE total mass (kg) $\left(r^{2}=0.564, P<0.05\right)$ found between evaporation rate and exposed body surface area $(\%)$ $\left(r^{2}=0.950, P<0.001\right)$ and between evaporation rate and perceived skin wetness $(\%)$ $\left(r^{2}=0.800, P<0.005\right.$, Figure 6$)$. In particular, the correlation between evaporation rate and exposed body surface area and that between evaporation and skin wetness were significant, showing the correlation coefficients of 0.974 and 0.895 , respectively.

\section{Discussion}

The purpose of the present study was to examine the firefighters' various subjective perceptions while wearing a wide range of PPE combinations. The data in NoJP and

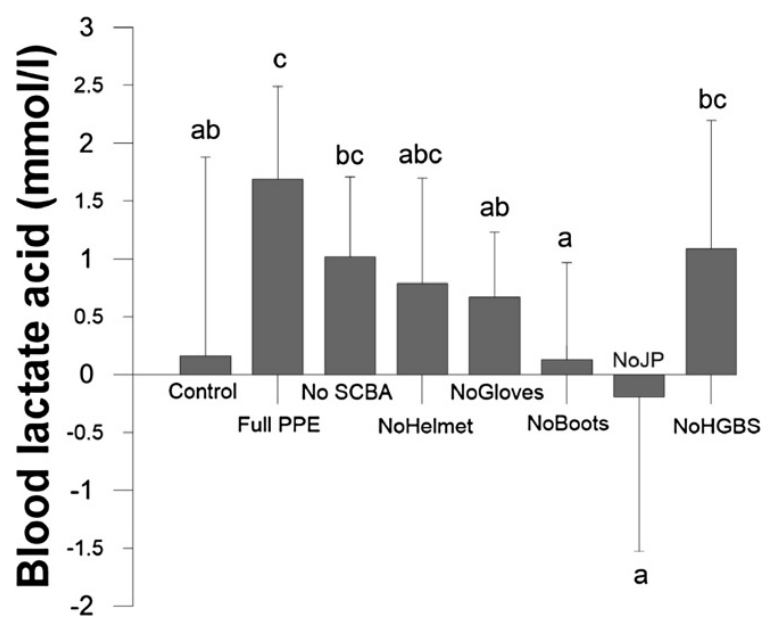

Figure 4 Changes in blood lactate concentration between before and after exercise. Different groups were distinguished by different letters. Groups are significantly different $(P=0.05)$ by Duncan post-hoc test $(P<0.05)$. 


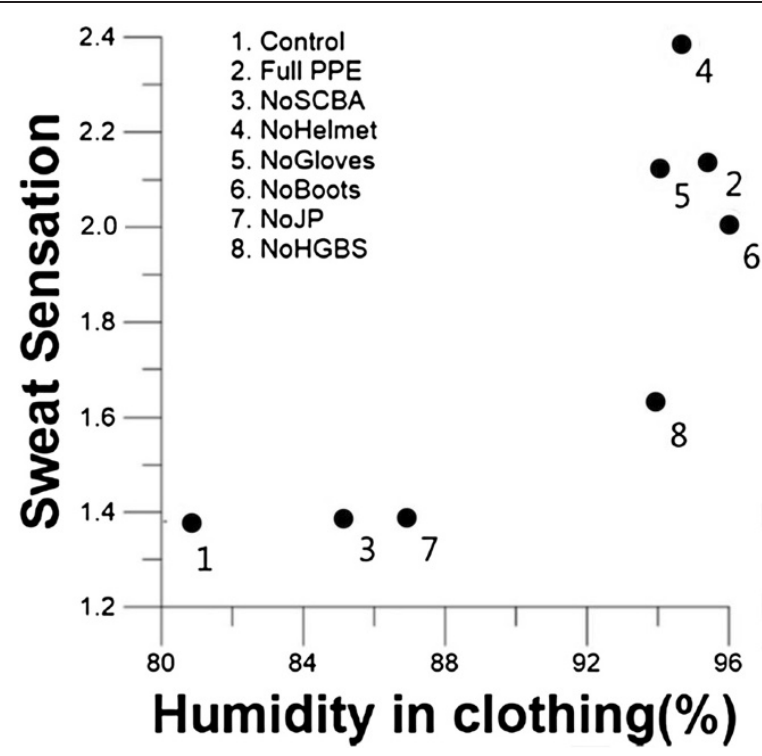

Figure 5 Relationship between sweat sensation and clothing microclimate humidity on the chest. Each number represents all different seven conditions of firefighters' gear and the control group. All data were the mean values of each condition at the end of exercise $(N=8)$. Y-axis represents sweat sensation; 1 (A little wet), 2 (Wet), and 3 (Very wet).

NoHGBS infer whole body exposure and whole peripheral body regions' exposure, respectively. The effect of total PPE weight was detected by comparison between NoSCBA and FullPPE. Likewise, the effect of exposing peripheral body regions could be explored by comparison among NoHelmet, NoGloves, and NoBoots. Although the present experiment did not have enough subjects to compare psychological factors, it was found that several significant differences and advantages or weakness of each condition exist.

\section{NoJP: benefit to improve overall subjective perceptions}

The NoJP condition reflects the influence of removing the heavy impermeable jacket and pants upon thermal ratings by comparing with FullPPE. Thermal sensation both in exercise and recovery and RPE were significantly lower than FullPPE. Sweat sensation in NoJP was also lower both in exercise and recovery than in FullPPE, though it was

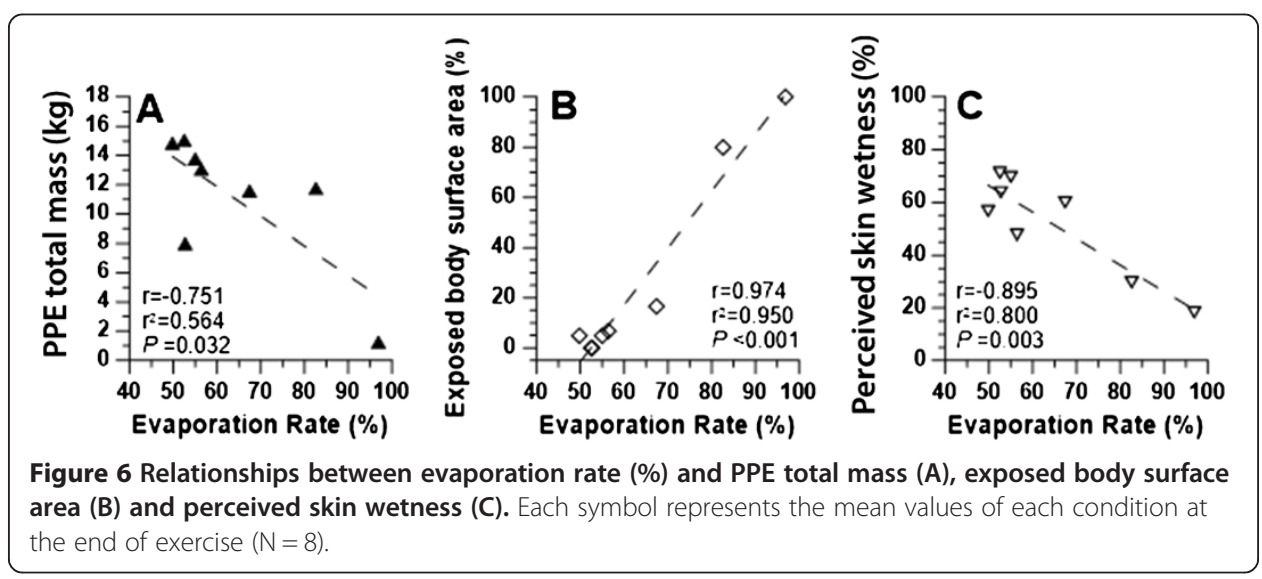


significant only in recovery. Overall, NoJP was the most effective ensemble to alleviate subjective burden with limited sweating compared to the other conditions. The present results showed that both factors (exposed BSA and PPE mass) have significantly strong correlations with evaporation rate (Figure 6). NoJP was the condition of which evaporation rate was the greatest among the seven PPE conditions.

Also, it would be interesting to discuss the blocking effect of heat dissipation from the peripheral body parts including the feet, hands, and head, through the comparison of NoJP and Control. Subjective perceptions including overall thermal sensation, sweat sensation, thermal comfort, thirst sensation, and RPE were not significantly different between NoJP and Control, while total sweat rate was 1.6 times greater in NoJP than in Control. These results indicate that subjective thermal strain increased by blocking the peripheral body parts and increasing clothing weight about $10 \mathrm{~kg}$.

\section{NoHGBS: benefit in recovery}

Overall thermal sensation in NoHGBS wavered between 'warm' and 'hot' during exercise, which was not significantly different with FullPPE, while it was recovered more quickly than in FullPPE. These results imply that exposing the head, hands, and feet could be greatly effective in relieving perceptual thermal strain in recovery after firefighting. According to (Koscheyev et al. 2008), heat flux through a liquid cooling garment was greater in the peripheral body parts than torso, which indicates that the most effective regions for heat release are the head, neck, hands, and feet rather than the torso.

At the scene of a fire, after firefighting, the best way to relieve heat strain is to remove all the firefighting gear, but it is not easy for firefighters to quickly doff their clothing and equipment (Kwon et al. 2012). Firefighters had problems with donning and doffing their gear, especially once it is wet (Barker et al. 2013). Also, firefighters are often required to re-enter structural fires following recovery periods of short duration around 15 min (Rayson et al. 2007). Furthermore, the periphery, such as the hands and feet contains arteriovenous anastomoses (AVAs) which control blood flow by shunting blood directly to the venous system from arterioles, bypassing capillaries. During heat stress, AVAs become maximally dilated allowing for greater blood flow to increase heat dissipation (Barr et al. 2010; Krogstad et al. 1995). Therefore, removing PPE covering peripheral regions in recovery could serve as an effective cooling strategy due to the increase of heat dissipation from the cutaneous blood supply in conditions where outdoor temperature is cooler than the skin temperature (Barr et al. 2010; Carter et al. 2007).

\section{NoBoots: benefit physiologically not psychologically}

The NoBoots condition was expected to be one of the most effective conditions, since the feet are known as one of the most inefficient body regions for load carriage (Lind and McNicol 1968; Munson 1901; Turrell and Robinson 1943). (Taylor et al. 2012) reported that body heat actively dissipates because of increased blood flow through bare feet. Heart rate and metabolic rate were significantly lower when not wearing protective boots rather than with the breathing apparatus. In the current study, RPE and blood lactate concentration in NoBoots were as low as those in NoJP and significantly lower than in FullPPE $(P<0.05)$, which supports the notion that exposing the feet is effective in relieving physiological burden. 
However, it seems that the NoBoots condition cannot effectively alleviate psychological burden. Sweat sensation, thermal sensation, and thermal comfort in NoBoots were not significantly different with FullPPE except for sweat sensation in the recovery period. In the present study, it was found that a relationship between sweat sensation and clothing humidity on the chest at the end of exercise was logarithmic. Over the clothing humidity of $95 \% \mathrm{RH}$ on the chest, subjects felt wet (score 2) or severe wet (over score 2). In NoBoots, clothing microclimate humidity on the chest had no significant superiority than those in FullPPE.

Nevertheless, it is interesting that the perceived wet area during exercise in NoBoots was $47.7 \%$ which was significantly smaller than FullPPE $(P<0.05)$. However, because the sweating regions tend to have great individual variations more studies are needed investigating differences in these areas.

\section{NoSCBA, NoHelmet, and NoGloves: less benefit to subjective perceptions}

Subjective perceptions in the conditions of NoSCBA, NoHelmet, and NoGloves were not significantly different with those in FullPPE. NoSCBA, representing mass reduction at the torso without any increase in exposed skin area could not alleviate metabolic rate (Taylor et al. 2012) but also subjective perceptions in the present study. But the microclimate humidity on the chest was significantly lower than FullPPE both at rest and in exercise. When considering that other supportive physiological factors, total sweat rate, didn't show any difference between in FullPPE and NoSCBA, shouldering the breathing apparatus could block disturbed convection inside the clothing. Significant difference of microclimate humidity didn't connect to significant difference of sweat sensation, which was supposed to be affected by insufficient number of subjects, since microclimate humidity on the chest and sweat sensation showed the tendency of logarithmic function as shown in Figure 5.

Meanwhile, it is quite interesting that NoHelmet and NoGloves showed no benefit in subjective responses since the head and hands are important body parts in terms of heat dissipation. In particular, the skin temperature of the head hardly changed even in the low temperature environment (Froese and Burton 1957; Sim 1996). Forehead temperature was maintained consistent around $30^{\circ} \mathrm{C}$ even in a cold environment $\left(15.6^{\circ} \mathrm{C}\right)$ without clothing, while skin temperature of extremities decreased substantially (Huizenga et al. 2004). Due to this gap between the head skin temperature and environmental temperature, heat is actively dissipated through exposed head skin by convection and radiation. However, in warm environments, dry heat dissipation is rarely evoked since skin temperatures of the extremities also approach the similar temperature of the environment. That is, heat dissipation through the head would be relatively less remarkable even though the head was exposed. This is the reason why effectiveness in alleviation of heat strain in NoHelmet was not found, and the same interpretation can be applied to NoGloves. Therefore, to effectively alleviate heat strain, it is required to expose several peripheral body parts simultaneously.

In a geometric sense, the head's globe form is not suited for heat dissipation, which is beneficial in cold environments. RPE in NoHelmet solely belonged to the same group as FullPPE, which represents how exhausted the subjects were. On the other hand, both hands and feet are more efficient in dissipating body heat in terms of the geometric form, since they have greater surface area compared to the volume. Nevertheless, 
NoGloves induced much worse subjective responses when compared to NoBoots. Such results could be related to the fact that protective gloves were lighter than that of protective boots, and hand motions are closer to the center of body mass when compared to the feet motion.

\section{Conclusion}

We assessed subjective perceptions among eight conditions consisting of seven conditions of firefighter's protective equipment and one control group. Thermal sensation, thermal comfort, sweat sensation, and RPE were analyzed in relation to clothing microclimate humidity, sweating responses, and blood lactate concentration. The significant findings from the present study were as follows:

First, the presence of boots affected the RPE or physiological factors rather than in thermal sensation and sweating responses. Specifically, blood lactate concentration after exercising in NoBoots was as low as in Control and NoJP, which implies that cooling the feet or reducing the mass of protective boots proved most effective for alleviating fatigue and exertion. This suggests that lighter boots or boots designed for easily donning and doffing would effectively reduce firefighters' metabolic burden.

Second, exposure of the hands and feet affected the local thermal sensation and sweat sensation of the hands and feet, respectively, while the efficacy of exposing the head was not found. This implies that the order of body part to cool down, which could be the hands or feet, but the head.

Third, a relationship between sweat sensation and clothing microclimate humidity on the chest at the end of exercise for the eight conditions shows a logarithmic function. Sweat sensation was expressed as 'wet' (score 2), when clothing microclimate humidity on the chest was over $95 \% \mathrm{RH}$, while sweat sensation could be controlled under 1.5, when clothing microclimate humidity on the chest was maintained at $95 \% \mathrm{RH}$ on average. Thus, it is important in reducing overall sweat sensation by effectively drying the chest during exercise and recovery periods.

Finally, heat loss by evaporation affects the alleviation of perceptual heat strain, and a relationship between evaporation rate and exposed skin area shows a strong linear function. Therefore improved design regarding the ease of removing equipment should be implemented, so that a higher exposed body surface area is reached as quickly as firefighting action ceases.

Competing interests

The authors declare that they have no competing interests.

\section{Authors' contributions}

SK participated in the experiment and carried out the data analysis and drafted the manuscript. YJJ participated in the experiment and discussion. YJB participated in the data analysis and discussion. JYL conceived of the study plan, participated in the experiment, the data anaylsis, and discussion, and authorized the total study. All authors read and approved the final manuscript.

\section{Author details}

${ }^{1}$ Department of Textiles, Merchandising and Fashion Design, Seoul National University, Seoul, Korea. ${ }^{2}$ Research Institute of Human Ecology, Seoul National University, Seoul, Korea. 
Barr, D, Gregson, W, Sutton, L, \& Reilly, T. (2009). A practical cooling strategy for reducing the physiological strain associated with firefighting activity in the heat. Ergonomics, 52(4), 413-420.

Barr, D, Gregson, W, \& Reilly, T. (2010). The thermal ergonomics of firefighting reviewed. Applied Ergonomics, 41, 161-172. Barr, D, Reilly, T, \& Gregson, W. (2011). The impact of different cooling modalities on the physiological responses in firefighters during strenuous work performed in high environmental temperatures. European Journal of Applied Physiology, 111, 959-967.

Blacker, SD, Fallowfield, JL, Bilzon, JL, \& Willems, ME. (2010). Neuromuscular function following prolonged load carriage on level and downhill gradients. Aviation, Space, and Environmental Medicine, 81, 745-753.

Bligh, J. (1973). Temperature regulation in mammals and other vertebrates. Amsterdam: North Holland Publishing Company.

Borg, GAV. (1982). Psychophysical bases of perceived exertion. Medicine and Science in Sports and Exercise, 14, 377-381.

Carter, JB, Rayson, M, Wilkinson, DM, Richmond, B, \& Blacker, S. (2007). Strategies to combat heat strain during and after firefighting. Journal of Thermal Biology, 32, 109-116.

Chou, C, Tochihara, Y, \& Kim, T. (2008). Physiological and subjective responses to cooling devices on firefighting protective clothing. European Journal of Applied Physiology, 104, 369-374.

Frank, SM, Raja, SN, Bulcao, CF, \& Goldstein, DS. (1998). Relative contribution of core and cutaneous temperatures to thermal comfort and autonomic responses in humans. Journal of Applied Physiology, 86, 1588-1593.

Froese, G, \& Burton, AC. (1957). Heat losses from the human head. Journal of Applied Physiology, 10, 235-241.

Gagge, AP, \& Gonzalez, RR. (1974). Physiological and physical factors associated with warm discomfort in sedentary man. Environmental Research, 7, 230-242.

Gagge, AP, Stolwijkm, AJ, \& Hardy, JD. (1967). Comfort and thermal sensations and associated physiological responses at various ambient temperatures. Environmental Research, 1, 1-20.

Gledhill, N, \& Jamnik, VK. (1992). Characterization of the physical demands of firefighting. Canadian Journal of Applied Sport Sciences, 17, 207-213.

Griefahn, B, Künemund, C, \& Bröde, P. (2003). Evaluation of performance and load in simulated rescue tasks for a novel design SCBA: effect of weight, volume and weight distribution. Applied Ergonomics, 34, 157-165.

Havenith, G, Holmer, I, \& Parsons, K. (2002). Personal factors in thermal comfort assessment: clothing properties and metabolic heat production. Energ Buildings, 34, 581-591.

Hooper, AJ, Crawford, JO, \& Thomas, D. (2001). An evaluation of physiological demands and comfort between the use of conventional and lightweight self-contained breathing apparatus. Applied Ergonomics, 32, 399-406.

Huizenga, C, Zhang, H, Arens, E, \& Wang, D. (2004). Skin and core temperature response to partial- and whole-body heating and cooling. Journal of Thermal Biology, 29, 549-558.

Knapik, JJ, Reynolds, KL, \& Harman, E. (2004). Soldier load carriage: historical, physiological, biomechanical, and medical aspects. Military Medicine, 169, 45-56.

Koscheyev, VS, Lee, JY, Kim, JH, Leon, GR, Kwon, S, \& Gernhardt, ML. (2008). Cooling and thermal strategies in the space suit for routine and emergency situations (SAE Technical Paper Series, Vol. Paper Number 2008 ICES-01-1993). Warrendale, PA: SAE International.

Krogstad, AL, Elam, M, Karlsson, T, \& Wallin, BG. (1995). Arteriovenous anastomoses and the thermoregulatory shift between cutaneous vasoconstrictor and vasodilator reflexes. Journal of the Autonomic Nervous System, 53, 215-222.

Kwon, JH, Lee, JS, Ha, GE, \& Kweon, SA. (2012). The importance and uncomfort degrees of the firefighters' active uniforms. Journal of Human Ecology, 16, 91-98.

Lee, JY, Choi, JW, \& Kim, H. (2008). Determination of body surface area and formulas to estimate body surface area using the alginate method. American Journal of Physical Anthropology, 27, 71-81.

Lee, JY, Bakri, I, Kim, JH, Son, SY, \& Tochihara, Y. (2013). The impact of firefighter personal protective equipment and treadmill protocol on maximal oxygen uptake. Journal of Occupational and Environmental Hygiene, 10, 397-407.

Lind, AR, Mcnicol, GW (1968) Cardiovascular responses to holding and carrying weights by hand and by shoulder harness. Journal of applied physiology, 25(3), 261-267.

McLellan, TM, Frim, J, \& Bell, DG. (1999). Efficacy of air and liquid cooling during light and heavy exercise while wearing NBC clothing. Aviation, Space, and Environmental Medicine, 70, 802-811.

Qu, X, \& Yeo, JC. (2011). Effects of load carriage and fatigue on gait characteristics. Journal of Biomechanics, 44, 1259-1263.

Rayson, M, Carter, J, Wilkninson, D, Richmond, V, \& Blacker, S. (2007). Recovery duration required prior to re-deployment during firefighting, search and rescue. Medicine and Science in Sports and Exercise, 35(5), S149.

Rossi, R. (2003). Fire fighting and its influence on the body. Ergonomics, 46, 1017-1033.

Sim, BJ. (1996). A Study on the variation of skin temperature on the adult male at environment temperature. Journal of the Ergonomics Society of Korea, 15, 71-88.

Son, SY, Lee, JY, \& Tochihara, Y. (2013). Occupational stress and strain in relation to personal protective equipment of Japanese firefighters assessed by a questionnaire. Industrial Health, 51, 214-222.

Taylor, NA, Lewis, MC, Notley, SR, \& Peoples, GE. (2012). A fractionation of the physiological burden of the personal protective equipment worn by firefighters. European Journal of Applied Physiology, 112(8), 2913-2921.

Turpin-Legendre, E, \& Meyer, JP. (2007). Comparison of physiological and subjective strains of two protective coveralls in two short physically simulated demanding tasks. Applied Ergonomics, 38, 249-252.

White, MK, Hodous, TK, \& Hudnall, JB. (1989). Physiological and subjective responses to working in disposable protective coveralls and repirators commonly used by the asbestos abatement industry. American Industrial Hygiene Association Journal, 50, 313-319.

Winslow, CEA, Herrington, LP, \& Gagge, AP. (1939). Physiological reactions and sensations of pleasantness under varying atmospheric conditions. ASHVE Trans, 44, 179-194.

doi:10.1186/s40691-014-0003-8

Cite this article as: Kim et al: Influences of partial components in firefighters' personal protective equipment on subjective perception. Fashion and Textiles 2014 1:3. 